\title{
Primary care summary of the British Thoracic Society Guideline on the management of non-cystic fibrosis bronchiectasis
}

\author{
*Adam T Hilla, Mark Pasteurb, Charles Cornford', Sally Welham d, Diana Bilton \\ a Department of Respiratory Medicine, Royal Infirmary and University of Edinburgh, Scotland, UK \\ ${ }^{\mathrm{b}}$ Department of Respiratory Medicine, Norfolk and Norwich University Hospital, Norwich, UK \\ c Fulcrum Medical Practice, Middlesbrough, UK \\ ' British Thoracic Society, London, UK \\ e Department of Respiratory Medicine, Royal Brompton Hospital, London, UK
}

Original submitted 9th July 2010; resubmitted 17th September 2010; revised version received 11th October 2010; accepted 22nd November 2010; online 18th February 2011

\begin{abstract}
The British Thoracic Society (BTS) has recently published a guideline for the management of non-cystic fibrosis (non-CF) bronchiectasis in children and adults. This paper summarises the key recommendations applicable to the primary care setting. The key points are:

- Think of the diagnosis of bronchiectasis in adults and children who present with a chronic productive cough or unexplained haemoptysis, and in children with asthma which responds poorly to treatment

- High resolution computed tomography (HRCT) scanning is needed to confirm the diagnosis

- Sputum culture should be obtained at the start of an exacerbation prior to initiating treatment with antibiotics. Treatment should be started whilst awaiting the sputum result and should be continued for 14 days

- Patients with bronchiectasis have significant morbidity. Management in primary care is aimed at improving morbidity, and includes patient education, treatment and monitoring, as well as appropriate referral to secondary care including assessment for long term antibiotics.

(C) 2011 Primary Care Respiratory Society UK. All rights reserved.

AT Hill et al. Prim Care Respir J 2011; 20(2): 135-140

doi:10.4104/pcrj.2011.00007
\end{abstract}

Keywords guideline, infection, bronchiectasis, BTS, summary, primary care, diagnosis, patient education, treatment, monitoring

\section{See linked editorial by Feldman on pg 107}

\section{Introduction}

Originally described by Laennec in 1819, bronchiectasis is a chronic, debilitating condition characterised by persistent cough, excessive sputum production and recurrent chest infections. Permanent abnormal dilatation of the airways contributes to impaired mucociliary clearance, which in turn leads to a vicious cycle of bacterial colonisation in normally sterile airways and excessive airways inflammation.

Determining the precise prevalence is difficult; the different diseases causing bronchiectasis vary across different countries and across different historical periods, and also the different techniques used to diagnose and define bronchietasis have changed; for example, modern high-resolution computed tomography (HRCT) scans can identify previously undiagnosed disease - though whether that represents clinically significant disease is debatable. As a guide, the average full-time GP with a list size of about 2,000 patients would expect to manage approximately two cases of bronchiectasis.

Bronchiectasis has been the subject of relatively few randomised controlled studies. The British Thoracic Society (BTS) guideline for the management of non-cystic fibrosis (non-CF) bronchiectasis has been published recently. ${ }^{1}$ This summary paper highlights the key guideline recommendations and also those issues within the guideline which are highly relevant to the

\footnotetext{
* Corresponding author: Dr Adam T Hill, Department of Respiratory Medicine, Edinburgh Royal Infirmary, 51 Little France Crescent, Edinburgh, EH16 4SA Scotland, UK. Tel: +44 (0)1312421921 Fax: +44 (0)1312421870 E-mail: adam.hill2@nhs.net
} 
AT Hill et al.

\section{Table 1. Key to evidence statements and grades of recommendations.}

\begin{tabular}{|c|c|}
\hline \multicolumn{2}{|c|}{ Levels of evidence } \\
\hline $1++$ & High quality meta-analyses, systematic reviews of RCTs, or RCTs with a very low risk of bias \\
\hline $1+$ & Well-conducted meta-analyses, systematic reviews, or RCTs with a low risk of bias \\
\hline $1-$ & Meta-analyses, systematic reviews, or RCTs with a high risk of bias \\
\hline \multirow[t]{2}{*}{$2++$} & High quality systematic reviews of case control or cohort studies \\
\hline & High quality case control or cohort studies with a very low risk of confounding or bias and a high probability that the relationship is causal \\
\hline $2+$ & $\begin{array}{l}\text { Well-conducted case control or cohort studies with a low risk of confounding or bias and a moderate probability that the } \\
\text { relationship is causal }\end{array}$ \\
\hline 2- & Case control or cohort studies with a high risk of confounding or bias and a significant risk that the relationship is not causal \\
\hline 3 & Non-analytic studies, e.g. case reports, case series \\
\hline 4 & Expert opinion \\
\hline \multicolumn{2}{|c|}{ Grades of recommendations } \\
\hline A & $\begin{array}{l}\text { At least one meta-analysis, systematic review, or RCT rated as } 1++ \text {, and directly applicable to the target population; or } \\
\text { A body of evidence consisting principally of studies rated as } 1+\text {, directly applicable to the target population, and demonstrating } \\
\text { overall consistency of results }\end{array}$ \\
\hline $\mathrm{B}$ & $\begin{array}{l}\text { A body of evidence including studies rated as } 2++ \text {, directly applicable to the target population, and demonstrating overall } \\
\text { consistency of results; or } \\
\text { Extrapolated evidence from studies rated as } 1++ \text { or } 1+\end{array}$ \\
\hline C & $\begin{array}{l}\text { A body of evidence including studies rated as } 2+\text {, directly applicable to the target population and demonstrating overall } \\
\text { consistency of results; or } \\
\text { Extrapolated evidence from studies rated as } 2++\end{array}$ \\
\hline $\mathrm{D}$ & $\begin{array}{l}\text { Evidence level } 3 \text { or } 4 \text {; or } \\
\text { Extrapolated evidence from studies rated as } 2+\end{array}$ \\
\hline Good & ractice points \\
\hline$\sqrt{ }$ & Recommended best practice based on the clinical experience of the guideline development group \\
\hline
\end{tabular}

management of bronchiectasis in primary care.

The levels of evidence and grades of recommendation used in the guideline ${ }^{1}$ are reproduced in Table 1.

\section{Background Aetiology}

The cause is post-infective in up to $42 \%$ of cases, ${ }^{2,3}$ but up to $50 \%$ of cases no underlying cause is identified. An underlying cause will be sought in secondary care as it may affect future management. In international series' the commonest causes identified include: post-infective (bacterial and viral pneumonia, pertussis or tuberculosis); immune defects; connective tissue disease; allergic bronchopulmonary aspergillosis; cystic fibrosis; ciliary defects; inflammatory bowel disease; obstruction or foreign body; aspiration or inhalation; asthma; congenital or airway abnormality.

\section{Symptoms}

Cough occurs in $>90 \%$. The cough is productive of sputum daily in $75-100 \%$ of cases, being mucopurulent or purulent in
$71-97 \%$ of cases. Other possible symptoms include dyspnoea, chest pain, haemoptysis, and the potential development of respiratory failure and cor pulmonale.

\section{Signs}

Signs include inspiratory crepitations (in about $70 \%$ of cases) and expiratory rhonchi (in about a third of adults). Finger clubbing is now rare.

\section{Prognosis and importance}

The effect on mortality is unclear. Decline in lung function may be gradual and it is not certain whether survival is adversely affected in the modern era, at least in developed countries. However, bronchiectasis has significant morbidity; it is associated with increased anxiety and depression scores, increased fatigue, and decreased quality of life. High depression scores are correlated with dyspnoea scores. Worse quality of life scores are associated with increased frequency of exacerbations and with Pseudomonas aeruginosa colonisation. Bronchiectasis (particularly the associated cough) can impact on family members. 


\section{Suspecting the diagnosis In children}

Particularly important presentations in primary care include chronic productive cough, or asthma responding poorly to treatment. Consider bronchiectasis in [recommendation level D]:

- Children who have a chronic moist/productive cough, especially between viral colds and lasting eight weeks or longer

- Asthma that does not respond to treatment

- Incomplete resolution of a severe pneumonia, or recurrent pneumonia

- Pertussis-like illness failing to resolve after six months

- Persistent and unexplained physical signs, especially persistent lung crackles

- Respiratory symptoms in children with structural or functional disorders of the oesophagus and upper respiratory tract

- Unexplained haemoptysis.

In adults

Chronic productive cough is the most common presentation, though this is also a common presentation of other common respiratory illnesses such as COPD. Think of bronchiectasis in [D]:

- Persistent productive cough particularly with the following:

o young age at presentation

o history of symptoms over many years

o absence of smoking history

o daily expectoration of large volumes of very purulent sputum

o haemoptysis

- Unexplained haemoptysis or non-productive cough (after other causes have been excluded)

- Patients thought to have COPD may have bronchiectasis alone or in addition. Consider bronchiectasis if:

o management is not straightforward

o there is slow recovery from lower respiratory tract infections

o there are recurrent exacerbations

o there is no history of smoking

- Sputum colonised with Pseudomonas aeruginosa.

\section{Investigations to be performed in primary care [D]}

- Chest X-ray (CXR). A normal chest X-ray does not exclude the diagnosis of bronchiectasis. In addition, an abnormal chest X-ray suggestive of bronchiectasis should not be regarded as diagnostic, and patients should not be coded as having bronchiectasis on the basis of a suggestive CXR

- HRCT scan of the chest is the gold standard investigation to establish the diagnosis of bronchiectasis. If HRCT is not available, then refer to secondary care [A]

- Spirometry in all adults and in children who are old enough (usually aged over 5 years)

- Sputum sample should be sent for microbiological analysis when clinically stable, and at the start of exacerbations before antibiotic treatment is initiated [D].

\section{Investigations in secondary care}

It is probable that everyone with a diagnosis of bronchiectasis will be referred to secondary care for an initial assessment which will include a search for underlying causes. The following will be undertaken in secondary care:

- HRCT chest $[\mathrm{A}]$

- Blood tests: FBC, ESR, CRP, Serum immunoglobulins $(G, A, M)$ and serum electrophoresis $[A]$. Serum $\lg E$, IgE to Aspergillus fumigatus or skin prick testing to Aspergillus fumigatus and Aspergillus precipitins [C]. This will identify patients with immunoglobulin deficiency and allergic bronchopulmonary aspergillosis

- Further immunological tests will be carried out if immunodeficiency suspected [D]

- Tests for cystic fibrosis if indicated [D]

- Sputum for routine microbiological culture (mycobacterial cultures in addition if indicated) [D].

\section{Follow-up of which patients in secondary care [D]?}

- All children with bronchiectasis

- Patients with chronic Pseudomonas aeruginosa, opportunist mycobacteria or MRSA colonisation

- Those patients with recurrent exacerbations ( $\geq 3$ per year)

- Deteriorating bronchiectasis with declining lung function

- Patients receiving prophylactic antibiotic therapy (oral or nebulised)

- Patients with bronchiectasis and associated rheumatoid arthritis [C], immune deficiency, inflammatory bowel disease and primary ciliary dyskinesia

- Allergic broncho-pulmonary aspergillosis

- Advanced disease, including those patients being considered for transplantation.

\section{The aims of treatment [D]}

- To identify and treat an underlying cause to prevent disease progression

- To maintain or improve pulmonary function

- To reduce exacerbations and improve health-related quality of life by reducing daily symptoms and exacerbations

- To achieve normal growth and development in children.

\section{Management options [D]}

- Patient education. Patients will need information about bronchiectasis, the different treatment approaches 
available and the rationale behind them. They should know how to recognise an exacerbation and how to access medical care early in exacerbations. The importance of producing a sputum sample should be made clear and the local procedures needed to process a sample explained. They should receive an individual plan for follow-up and monitoring (either within primary care or with secondary care)

- Primary care nurses could receive training in the management of bronchiectasis

- Physiotherapy. All patients should be assessed by a specialist chest physiotherapist for teaching of airway clearance techniques and exercise [D]

- All patients should have an annual influenza vaccination and 5-yearly pneumococcal vaccination [B]

- Assess patients with airflow obstruction for reversibility to $\beta_{2}$-agonist and anti-cholinergic bronchodilators. Institute maintenance therapy where lung function or symptoms improve on therapy [D]

- Inhaled steroids should not be used routinely in children or adults with bronchiectasis (unless the patient has additional asthma or co-existing COPD and meets the criteria for inhaled steroids as recommended by international guidelines) [D]

- The clinical efficacy of carbocysteine and mannitol has not been demonstrated in randomised controlled trials to date but can be tried on an individual basis if the clinical need arises [D]

- There is no evidence of a role for leukotriene receptor antagonists, other anti-inflammatory drugs, or methylxanthines [D]

- The role of long term antibiotics, surgery and management of major haemoptysis - see below

- The indications for long term oxygen therapy are the same as for COPD [D]. The additional role of non-invasive ventilation [NIV] is discussed below.

\section{Monitoring bronchiectasis patients in primary care}

- Assessment of bronchiectasis patients' symptoms should include a record of both sputum purulence and estimated or measured 24-hour sputum volume when clinically stable [D]. The number of infective exacerbations per annum should be noted, including the frequency and nature of antibiotic usage [D]

- Sputum should be sent for microbiological analysis when the patient is clinically stable, and should be sent at the start of an exacerbation before antibiotic treatment is started [D]

- Repeat assessment of forced expiratory volume in one second ( $\left.F E V_{1}\right)$, forced vital capacity (FVC) and peak expiratory flow (PEF) should be made at least annually in those patients attending secondary care [D]: consider annual spirometry in patients managed solely in primary care

- Repeat chest X-rays need only be done if clinically indicated [D]

\section{Management of exacerbations}

Is it an exacerbation, and when are antibiotics required?

- Daily symptoms of cough and sputum production are frequent, and more severe bronchiectasis patients often expectorate mucopurulent or purulent sputum and culture respiratory pathogens when they are apparently clinically stable. This is more common in adults

- The presence of mucopurulent or purulent sputum alone, or the isolation of a pathogen alone, is not necessarily an indication for antibiotic treatment

- Antibiotics are recommended for exacerbations that present with an acute deterioration (usually over several days) with worsening local symptoms (cough, increased sputum volume or change of viscosity, increased sputum purulence \pm increasing wheeze, breathlessness, haemoptysis) and/or systemic upset.

\section{Should the patient be admitted to hospital?}

Children, Inpatient treatment is recommended for the following:

- Breathlessness with a raised respiratory rate and increased work of breathing

- Circulatory failure, respiratory failure or cyanosis

- Temperature $\geq 38^{\circ} \mathrm{C}$

- Patient unable to take oral therapy

- Intravenous therapy required in patients with clinical failure after oral antibiotics. Even if community services can arrange this in the patient's home, refer to secondary care for advice and management

Adults. Inpatient treatment is recommended for the following:

- Development of cyanosis or confusion

- Breathlessness with a respiratory rate $>25$ /minute

- Circulatory failure, respiratory failure, cyanosis or confusion

- Temperature $\geq 38^{\circ} \mathrm{C}$

- Patient unable to take oral therapy

- Patient unable to cope at home

- Intravenous therapy required in patients with clinical failure after oral antibiotics. Even if community services can arrange this in the patient's home, refer to secondary care for advice and management

\section{If treatment is in primary care, what should one assess?}

- History

- Clinical examination 
- Sputum culture, preferably before treatment

- Adults. Specimens should ideally reach the microbiology laboratory within three hours to maximise microbiology yield, though in remote areas, posting the sample and processing within 24 hours may be acceptable

- Obtain from children if possible - spontaneous sputum if present, if not perform a throat swab. Caution should be taken in interpreting these cultures because many of the pathogens may also be normal upper airway commensals.

If treatment is in primary care, what factors should one consider regarding antibiotic treatment? For both children and adults:

- Empirical antibiotics should be started whilst awaiting sputum microbiology [D]. Previous sputum bacteriology results can be useful in deciding which antibiotic to use [D]. In general, antibiotic courses for 14 days are standard

- Antibiotics can be modified subsequently once the pathogen is isolated only if there is no clinical improvement and the treatment should then be guided by antibiotic sensitivity results [D]. Some patients may respond to antibiotic treatment despite resistance to that drug in vitro

- Children and adults not responding to empirical antibiotics courses should have an organism identified by spontaneous sputum if present; if not, refer to secondary care [D]

- Intravenous antibiotics should be considered when patients are unwell, have resistant organisms, or have failed to respond to oral therapy (this is most likely to apply to patients with Pseudomonas aeruginosa) - refer to secondary care $[C]$

\section{Children}

- If there is no previous bacteriology, the first line treatment is amoxicillin, or clarithromycin for patients who are penicillin allergic (see the British National Formulary for Children for the dosage. ${ }^{4} \mathrm{NB}$, use the doses specified for "severe infection")

\section{Adults}

- If there is no previous bacteriology, first line treatment is amoxicillin $500 \mathrm{mg}$ three times daily $[\mathrm{B}]$, or clarithromycin $500 \mathrm{mg}$ twice daily for patients who are penicillin allergic [C]

- High dose oral regimens - e.g. amoxicillin $1 \mathrm{gm}$ three times daily or amoxicillin 3gm twice daily - may be needed in patients with severe bronchiectasis chronically colonised with Haemophilus influenzae [B]

- Ciprofloxacin should be used in patients colonised with Pseudomonas aeruginosa, with cautious use in the elderly [B]

- There is no evidence to support the routine use of antiviral drugs in exacerbations [D].

\section{Long-term antibiotic treatment}

- Long-term antibiotics should be considered in all children with frequent symptoms or severe disease, and in adults experiencing $\geq 3$ exacerbations per year requiring antibiotic therapy or with fewer exacerbations causing significant morbidity [C]

- All patients in whom long-term antibiotics are being considered need referral to secondary care

- Where possible, long-term antibiotic regimens should be determined by prior respiratory microbiology in children and by sputum microbiology in adults when clinically stable [D]

- Long-term use of oral quinolones should be avoided in children [D] and in adults [C] until further studies are available

- Macrolides may have disease modifying activity in adults, and preliminary data suggest the need for a large randomised controlled trial [C]

- For adults, high doses should not be used initially in order to minimise side effects [C]

- Long-term antibiotics may result in antibiotic resistance in individual patients, and alternative antibiotics should be chosen dependent on sensitivity results [D]

- The role of nebulised antibiotics long-term would be determined by secondary care. This would be more likely for children with frequent recurrent exacerbations (or deteriorating bronchiectasis) despite long-term oral antibiotics or if oral antibiotic therapy is not appropriate [D], and in both adults and children who meet the criteria for long-term antibiotics and who also have chronic Pseudomonas aeruginosa colonisation. Commonly used agents are gentamicin, tobramycin and colomycin, but all are unlicensed [D]

- Randomised controlled trials are needed to investigate the efficacy of nebulised antibiotics both in children and adults with bronchiectasis chronically colonised with organisms other than Pseudomonas aeruginosa [D]

\section{Managing massive haemoptysis (>25mls/day)}

- Admit urgently to hospital [D]

- Bronchial artery embolisation and/or surgery is first line therapy for the management of massive haemoptysis [D]

\section{Other treatment options}

- Lung resection surgery may be considered in patients with localised disease in whom symptoms are not controlled by medical treatment (after being reviewed first by a chest physician) [D]

- Non-invasive ventilation (NIV) can improve quality of life in some patients with chronic respiratory failure due to bronchiectasis [D]. Evidence for survival benefit is lacking, though some patients are successfully treated with NIV for significant lengths of time which may reduce hospitalisations [D]. 


\section{Audit criteria}

The audit criteria included in the guideline ${ }^{1}$ are as follows:

- All patients should be assessed for underlying cause(s)

- Sputum microbiology should be checked prior to antibiotics being given for exacerbations

These criteria have been developed into a more detailed audit tool for the management of bronchiectasis in secondary care; this is available via the BTS audit programme at https://audits.brit-thoracic.org.uk/. A statement of audit standards based on the BTS guideline is also available from this website.

\section{Conclusions}

This summary of the first UK national guideline for the management of non-CF bronchiectasis in adults and children provides guidance for those in primary care on the background, clinical assessment and investigation of patients, the principles of and the broad approach to management, the use of antibiotics, and the management of advanced disease. It has identified significant areas for research which we hope will be addressed by large trials to inform future guidelines. The aim is that the guideline, ${ }^{1}$ and this primary care-focussed summary, will promote a level of knowledge and care in both primary and secondary care to match that of asthma and COPD

\section{Acknowledgements}

We would like to thank the members of the Guideline group:

Professor Andrew Bush, Dr Steven Cunningham, Dr Xavier Emmanuel

Jane French, Dr Mike Greenstone, Professor David M Hansell, Alex Harvey, Dr Richard Herriot, Karen Heslop, Dr Pota Kalima, Frances Sinfield, Dr Samantha Sonnappa, Dr David A Spencer, Professor Robert A Stockley, Lorna Willcox, Dr Robert Wilson, Mr G Wyn Parry.

The assistance of Julia Bott, Jennifer Pryor and Dr Colin Wallis is also gratefully acknowledged.

We would also like to thank Dr John White, Chairman, BTS Standards of Care Committee, and Professor Wisia Wedzicha, former Editor-in-Chief, Thorax.

\section{Conflicts of interest}

None

\section{References}

1. Pasteur MC, Bilton D, Hill AT. British Thoracic Society guideline for non cystic fibrosis bronchiectasis. Thorax 2010;65:Suppl 1.

2. Nicotra MB, Rivera M, Dale AM, et al. Clinical, pathophysiologic, and microbiologic characterization of bronchiectasis in an aging cohort. Chest 1995;108:955-61. http://dx.doi.org/10.1378/chest.108.4.955

3. Pasteur MC, Helliwell SM, Houghton SJ, et al. An investigation into causative factors in patients with bronchiectasis. Am J Respir Crit Care Med 2000;162(4 Pt 1):1277-84.

4. British National Formula for children 2009: http://bnfc.org/.

\section{IPCRG membership - could you follow their lead?}

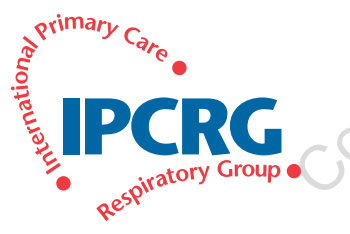

The IPCRG supports the development of new national primary care groups with a shared interest in respiratory care.

They can then join us as associate members, and once established, as full members.
Here are some recent activities from our membership:

In early May GRAP, the Spanish group held its second meeting in Barcelona with more than 90 attendees from all over Spain. Highlights included multidisciplinary presentations on informatics to support disease management and patientcentred care, new pharmacological evidence and new tests for pulmonary function. GRAP is also involved in producing innovative Spanish phenotype-based guidelines on COPD management (gesEPOC) due to be launched very soon.

The same weekend, 150 primary care professionals from our Nordic groups attended a joint Nordic conference.

To observe World Asthma Day 201 1, our Associate Member in Kolkata, the Indian Primary Respiratory Care \& Allergy Foundation (IPRCAF), organised a walk for about 400 school pupils, staff at one of the local health clinics and members of Lions Clubs International. There were also medical education sessions for over 100 doctors and discussions about the burden of asthma, air pollution and urgent need for appropriate public education to alleviate the debilitating fear of an asthma diagnosis and using an inhaler.

Our Dutch group, CAHAG, is helping deliver a European Respiratory Society Primary Care Day on Saturday 24 September at which many IPCRG colleagues are speaking. You can view the programme in this issue of the PCRJ on page 226. Do go!

To find out more about membership, contact our Business Manager, Sam Louw BusinessManager@theipcrg.org and visit our website: http://www.theipcrg.org/members/index.php 